\title{
Association of Neutrophil Extracellular Traps with the Development of Idiopathic Osteonecrosis of the Femoral Head
}

Mayu Nonokawa, ${ }^{*}$ Tomohiro Shimizu, ${ }^{\dagger}$ Miku Yoshinari, ${ }^{*}$ Yamato Hashimoto, ${ }^{*}$ Yusuke Nakamura, ${ }^{*}$ Daisuke Takahashi, Tsuyoshi Asano, ${ }^{\dagger}$ Yuka Nishibata, ${ }^{*}$ Sakiko Masuda, * Daigo Nakazawa, ${ }^{\ddagger}$ Satoshi Tanaka, ${ }^{\S}$ Utano Tomaru, ${ }^{\top}$ Norimasa Iwasaki, and Akihiro Ishizu*

From the Department of Medical Laboratory Science, ${ }^{*}$ Faculty of Health Sciences, and the Departments of Orthopedic Surgery, ${ }^{\dagger}$ Rheumatology, Endocrinology and Nephrology, ${ }^{\ddagger}$ and Pathology, ${ }^{\mathbb{}}$ Faculty of Medicine and Graduate School of Medicine, Hokkaido University, Sapporo; and the Center for Cause of Death Investigation, ${ }^{\$}$ Faculty of Medicine, Hokkaido University, Sapporo, Japan

Accepted for publication

July $9,2020$.

Address correspondence to Akihiro Ishizu, M.D., Ph.D., Department of Medical Laboratory Science, Faculty of Health Sciences, Hokkaido University, Kita-12, Nishi-5, Kita-ku, Sapporo 060-0812, Japan. E-mail: aishizu@med. hokudai.ac.jp.

\begin{abstract}
Idiopathic osteonecrosis of the femoral head $(\mathrm{ONFH})$ is defined as necrosis of osteocytes due to a nontraumatic ischemia of the femoral head. Iatrogenic glucocorticoid administration and habitual alcohol intake are regarded as risk factors. It has been suggested that glucocorticoid-induced activation of platelets contributes to the local blood flow disturbance of the femoral head. Both activated platelets and alcohol can induce neutrophil extracellular traps (NETs). To determine the association of NETs with the development of idiopathic $\mathrm{ONFH}$, surgically resected femoral heads of patients with idiopathic ONFH and osteoarthritis were assessed for existence of NET-forming neutrophils by immunofluorescence staining. NET-forming neutrophils were present in small vessels surrounding the femoral head of patients with idiopathic ONFH but not osteoarthritis. Moreover, Wistar-Kyoto rats were intravenously injected with NET-forming neutrophils or neutrophils without NET induction, and then the ischemic state of the tissue around the femoral head was evaluated by immunohistochemistry for hypoxiainducible factor- $1 \alpha$. NET-forming neutrophils circulated into the tissue around the femoral head, and hypoxia-inducible factor- $1 \alpha$ expression in the tissue was higher compared with that of rats intravenously administered with neutrophils without NET induction. Furthermore, ischemic change of osteocytes was observed in the femoral head of rats given an i.v. injection of NET-forming neutrophils. The collective findings suggest that NETs are possibly associated with the development of idiopathic ONFH. (Am J Pathol 2020, 190: 2282-2289; https://doi.org/10.1016/j.ajpath.2020.07.008)
\end{abstract}

Idiopathic osteonecrosis of the femoral head $(\mathrm{ONFH})$ is defined as necrosis of osteocytes due to a non-traumatic ischemia of the femoral head. ${ }^{1}$ It is a refractory disease that causes a collapse of the femoral head and a dysfunction of the hip joint, resulting in a decline in quality of life. Although the pathogenesis has not been revealed, it is well known that iatrogenic glucocorticoid administration and habitual alcohol intake are the risk factors for idiopathic $\mathrm{ONFH}^{2}$ A previous survey has demonstrated that, in patients with idiopathic ONFH, $51 \%$ were associated with glucocorticoids, $31 \%$ with alcohol, and $3 \%$ with both.

Recent studies have suggested that, in addition to a certain genetic background, insufficient blood supply to the femoral head, influence of lipid metabolism, decrease in osteogenesis, and apoptosis of osteocytes - all these can be caused by glucocorticoids-are related to glucocorticoidassociated ONFH. ${ }^{4}$ The contribution of platelet activation induced by glucocorticoids to the circulatory disorder of the femoral head has been pointed out. ${ }^{5}$ In contrast, it has been shown that an excessive consumption of alcohol reduces the differentiation ability of osteoblasts. ${ }^{6}$ As all patients who abuse alcohol do not develop ONFH, it is considered that a

Supported by an Uehara Memorial Foundation grant (T.S.).

Disclosures: None declared. 
certain genetic factor is also involved in the susceptibility of alcohol-associated ONFH.

Neutrophil extracellular trap (NET) is a web-like structure released from activated neutrophils, which consists of unraveled DNA accompanied by cytoplasmic antimicrobial proteins. ${ }^{8}$ NETs can trap microorganisms by the web-like DNA, kill them by antimicrobial proteins, and consequently prevent the host from spreading the microbes. Although NETs play an essential role in innate immunity, an excessive formation of NETs can be a cause of microangiopathy. ${ }^{9}$ Both activated platelets and alcohol can induce NETs. ${ }^{10,11}$ Thus, we have hypothesized that if there are NET-forming neutrophils-defined as neutrophils in the process of NET release-in small vessels surrounding the femoral head, these cells can be associated with the development of idiopathic ONFH.

In this study, we first examined the existence of NET-forming neutrophils in small vessels surrounding the femoral head of idiopathic ONFH and osteoarthritis (OA) as a disease control by immunofluorescence staining. As expected, NET-forming neutrophils were present in small vessels surrounding the femoral head of idiopathic ONFH but not of OA. Next, NET-forming neutrophils were injected intravenously into rats to determine whether NETs in small vessels surrounding the femoral head can induce ischemia of the tissue. It has been shown that NETs are degraded by serum factors, including DNase $\mathrm{I},{ }^{12}$ and that serum DNase I activity is influenced by aging. ${ }^{13}$ Thus, before conducting i.v. injection, the serum ability of NET degradation between young adult (4-week-old) and senior aged (24-week-old) rats were compared. Interestingly, serum NET degradation ability was significantly lower in senior aged rats than in young adult rats. On the basis of these findings, NET-forming neutrophils were injected intravenously into senior aged rats. This study demonstrates that intravenously injected NET-forming neutrophils circulated into the tissue around the femoral head and suggests that this event can induce ischemia of the femoral head.

\section{Materials and Methods}

\section{Human Subjects}

The femoral heads of patients (50 to 87 years old), which were surgically resected with total hip arthroplasty from April 2018 to October 2019 at the Department of Orthopedic Surgery, Hokkaido University Hospital, were used in this study. These femoral heads were derived from 14 idiopathic ONFH patients (50 to 86 years old; 6 females) and 12 OA patients (52 to 87 years old; 7 females). The age and sex variance was not statistically different between idiopathic ONFH and OA patients (Table 1). Among the 14 idiopathic ONFH patients, 4 were associated with glucocorticoid medication, 2 were associated with alcohol abuse, and 8 were associated with both (Supplemental Table S1). Underlying diseases that required glucocorticoid treatment included ulcerative colitis, bullous pemphigus, chronic inflammatory demyelinating polyneuropathy, pulmonary aspergillosis, systemic sclerosis, rheumatoid arthritis, drug-induced hypersensitivity syndrome, eosinophilic granulomatosis with polyangiitis, graft-versus-host disease after bone marrow transplantation, polymyositis, IgA nephritis, and rheumatic interstitial pneumonitis.

Written informed consent was obtained from all the patients. This study was approved by the Ethics Committee of Hokkaido University Hospital (approval number 018-0075).

\section{Pathologic Diagnosis}

The resected femoral heads were fixed with $10 \%$ formalin and then decalcified in 10\% EDTA-2Na solution. Several months later, the presence or absence of necrosis in the coronary section of the femoral head was determined macroscopically. Thereafter, macroscopic diagnosis was confirmed microscopically.

\section{Immunofluorescence Staining for NET-Forming Neutrophils}

To determine the presence of NET-forming neutrophils in small vessels surrounding the femoral head, the connective tissue containing the synovial membrane appended to the femoral head was sampled before decalcification. The formalin-fixed connective tissue samples were then embedded in paraffin. These formalin-fixed, paraffinembedded tissues were sliced into sections ( $4 \mu \mathrm{m}$ thick) and then deparaffinized with xylene. The deparaffinized sections were autoclaved at $121^{\circ} \mathrm{C}$ for 20 minutes with Tris-EDTA buffer ( $\mathrm{pH}$ 9.0) to retrieve antigens and then soaked in Protein Block Serum-Free (Dako, Glostrup, Hovedstaden, Denmark) for 10 minutes to avoid the non-specific reaction of antibodies. Thereafter, the sections were made to react first with goat anti-human myeloperoxidase (MPO) antibody (1:200 dilution; R\&D Systems, Minneapolis, MN) overnight at $4{ }^{\circ} \mathrm{C}$ and next with rabbit anti-human citrullinated histone H3 antibody (1:100 dilution; Abcam, Cambridge, MA) as a NET marker ${ }^{14}$ for 60 minutes at room temperature. The sections were then allowed to react with Alexa Fluor 488-conjugated donkey anti-goat IgG antibody (1:500 dilution; Abcam) and Alexa Fluor 594-conjugated donkey anti-rabbit IgG antibody (1:500 dilution; Abcam) for 60 minutes in the dark at room temperature. Finally, the sections were mounted with VECTASHIELD Mounting Medium with DAPI (Vector Laboratories, Burlingame, CA) and observed under a fluorescence microscope.

\section{Rats}

Male Wistar Kyoto (WKY) rats (young adult, 4 weeks old; senior aged, 24 weeks old) were purchased from Sankyo Laboratory (Sapporo, Japan). Experiments using rats were performed in accordance with the Guidelines for the Care 
Table 1 Patients Enrolled in This Study

\begin{tabular}{llll}
\hline & Idiopathic & & \\
Demographics & ONFH $(n=14)$ & OA $(n=12)$ & $P$ value \\
\hline Age, mean \pm SD, years & $61.8 \pm 9.3$ & $68.1 \pm 9.4$ & 0.11 \\
Sex, F:M ratio & $6: 8$ & $7: 5$ & 0.23 \\
\hline
\end{tabular}

F, female; M, male; $\mathrm{OA}$, osteoarthritis; $\mathrm{ONFH}$, osteonecrosis of the femoral head.

and Use of Laboratory Animals at Hokkaido University (permission number 15-0034).

\section{Measurement of NET Degradation Ability of Rat Serum}

Peripheral blood neutrophils from healthy volunteers were seeded in the wells of four-well chamber slides $\left(1 \times 10^{6}\right.$ cells $/ \mathrm{mL}$ ). The cells were exposed to $100 \mathrm{nmol} / \mathrm{L}$ phorbol 12-myristate 13-acetate (PMA; Sigma-Aldrich, St. Louis, MO) for 4 hours at $37^{\circ} \mathrm{C}$ to induce NETs. After washing with phosphate-buffered saline (PBS), the samples were incubated in $10 \%$ serum of young adult (4-week-old) WKY rats $(n=5)$ and senior aged (24-week-old) WKY rats $(n=5)$ for 30 minutes at $37^{\circ} \mathrm{C}$. Thereafter, the samples were washed with PBS and then fixed with $4 \%$ paraformaldehyde for 15 minutes at room temperature. After washing with PBS, the remaining samples were mounted with the DAPI-containing solution. Photomicrographs (magnification, $\times 200$ ) were taken randomly (six fields per well). The DAPI-positive area was measured using ImageJ software version 1.50i (NIH, Bethesda, MD; https://imagej. nih.gov/ij), and the NET degradation rate (percentage) was calculated as described elsewhere. ${ }^{15}$ The use of human peripheral blood was permitted by the Ethics Committee of the Faculty of Health Sciences, Hokkaido University (permission number 18-34).

\section{Collection of Neutrophils from the Abdominal Cavity of Rats}

WKY rats (4 weeks old) were given an i.p. injection of $3 \%$ thioglycolate (Becton Dickinson, Franklin Lakes, NJ) to induce peritonitis. After 48 hours, inflammatory cells infiltrating into the peritoneum were collected by flushing the abdominal cavity with - and then resuspending the washedout cells in-PBS containing $1 \mathrm{mmol} / \mathrm{L}$ EDTA. Neutrophils composed $>90 \%$ of the collected cells.

\section{Induction of NETs and Collection of NET-Forming Neutrophils}

The collected cells were resuspended in RPMI 1640 medium supplemented with $10 \%$ fetal bovine serum and then seeded in 10-cm culture dishes $\left(1 \times 10^{6}\right.$ cells $\left./ \mathrm{mL}\right)$. The cells were then exposed to $100 \mathrm{nmol} / \mathrm{L}$ PMA for 4 hours at $37^{\circ} \mathrm{C}$ to induce NETs. After washing with PBS, the substance that remained on the dishes, which contained abundant NET-forming neutrophils, was collected using a cell scraper and then resuspended in PBS.

\section{Injection of NET-Forming Neutrophils into Rats}

The substance that contained abundant NET-forming neutrophils was injected into senior aged (24-week-old) WKY rats (group $1 ; n=6$ ) via the tail vein (substance derived from $2 \times 10^{7}$ cells $/ \mathrm{kg}$ recipient weight) twice with 1 -week
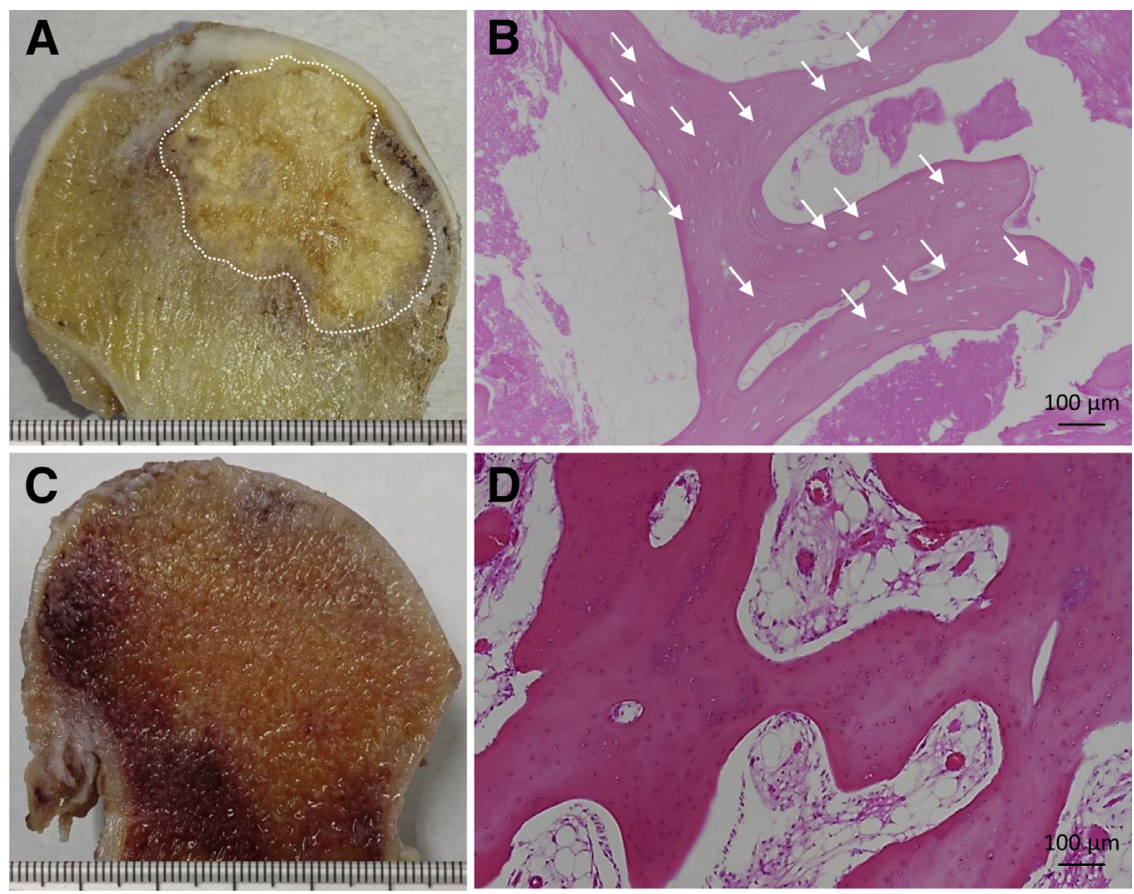

Figure 1 Representative macroscopic (A and C) and microscopic (B and $\mathbf{D}$ ) findings of the coronary section of the femoral head showing idiopathic osteonecrosis of the femoral head (A and $\mathbf{B}$ ) and osteoarthritis (C and $\mathbf{D})$. The area enclosed by the dotted line exhibits necrosis. White arrows indicate osteocytes with disappeared nuclei. Scale bars $=100 \mu \mathrm{m}(\mathbf{B}$ and $\mathbf{D})$. 

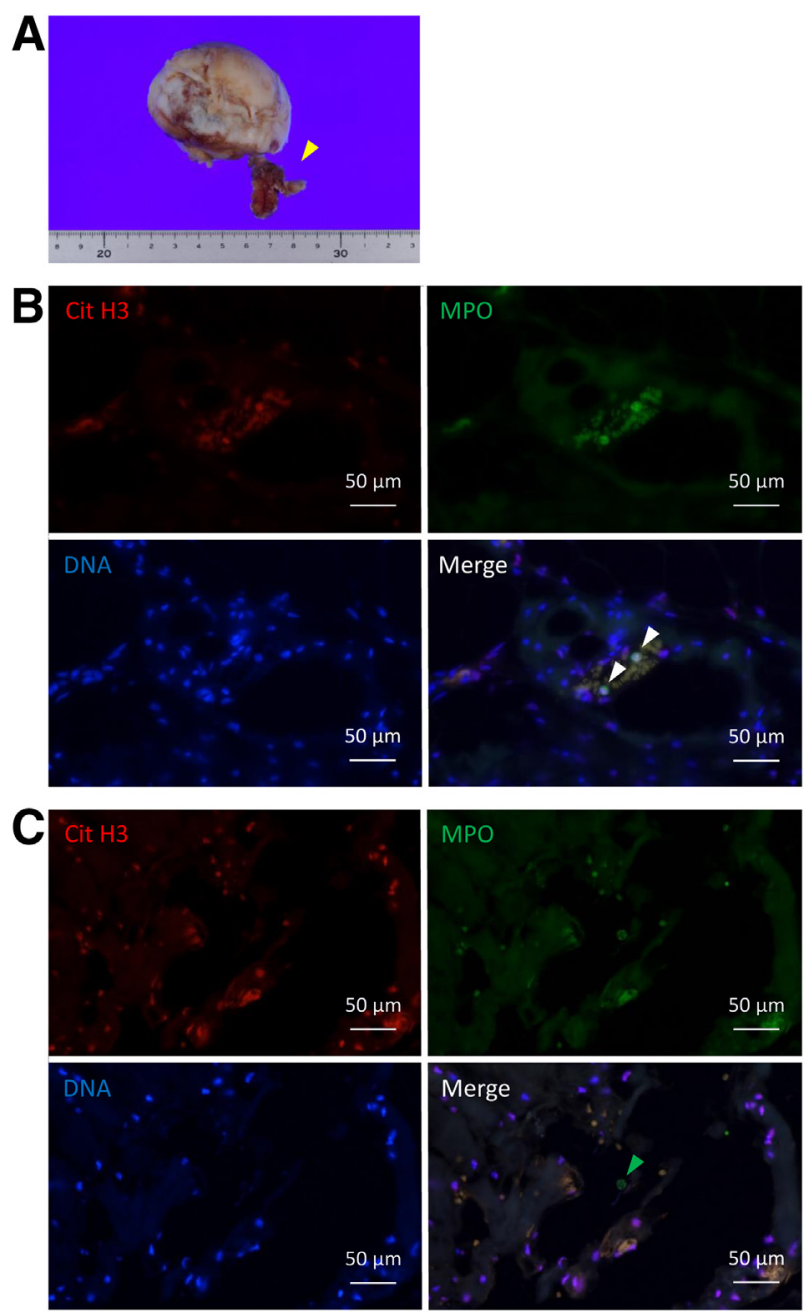

Figure 2 Neutrophil extracellular trap (NET)-forming neutrophils in small vessels surrounding the femoral head. A: Connective tissue containing the synovial membrane appended to the femoral head (arrowhead), which was subjected to immunofluorescence staining. B: Representative findings of NET-forming neutrophils (arrowheads) in the tissue around the femoral head showing idiopathic osteonecrosis of the femoral head. C: Representative findings of the tissue around the femoral head showing osteoarthritis. A myeloperoxidase-positive $\left(\mathrm{MPO}^{+}\right)$cell (arrowhead) but not a NET-forming neutrophil is observed. Scale bars $=50 \mu \mathrm{m}$ (B and $\mathbf{C})$. Cit $\mathrm{H} 3$, citrullinated histone $\mathrm{H} 3$.

interval. As controls, the cells collected from the abdominal cavity of rats with peritonitis were similarly injected into senior aged (24-week-old) WKY rats (group 2; $n=4$; $2 \times 10^{7}$ cells/kg recipient weight) without NET induction. One week after the last injection, all rats were euthanized for histopathologic evaluation.

\section{Histopathologic Evaluation}

The systemic organs and the connective tissue surrounding the femoral head were resected. The formalin-fixed, paraffin-embedded tissues were cut into sections $(4 \mu \mathrm{m}$ thick), deparaffinized with xylene, and then subjected to hematoxylin and eosin staining and elastica-van Gieson staining. The sections of the connective tissue surrounding the femoral head were also subjected to immunofluorescence staining for NETs and immunohistochemistry for hypoxia-inducible factor $1 \alpha$ (HIF-1 $1 \alpha)$. Immunohistochemistry for HIF-1 $\alpha$ was done as follows: formalin-fixed, paraffin-embedded sections were autoclaved at $121^{\circ} \mathrm{C}$ for 10 minutes with Tris-EDTA buffer $(\mathrm{pH} \mathrm{9.0)}$ to retrieve antigens and then exposed to methanol supplemented with $3 \%$ hydrogen peroxide for 10 minutes to consume endogenous peroxidase in the tissue. After blocking the nonspecific reaction of antibodies with $10 \%$ goat serum, the sections were made to react with mouse anti-human HIF-1 $\alpha$ antibody (1:800 dilution; Abcam), which can cross-react with rat HIF- $1 \alpha$, for 60 minutes at room temperature. After washing out unbound antibodies with PBS, the sections were next allowed to react with horseradish peroxidase-conjugated goat anti-mouse IgG antibody (1:50 dilution; Abcam) for 60 minutes at room temperature. Horseradish peroxidase activity was developed with diaminobenzidine.

\section{Statistical Analysis}

The $\chi^{2}$ test was applied for the comparison of the difference in frequency between two groups. The $U$-test was applied for the comparison of values between two nonparametric groups. $P<0.05$ was regarded as statistically significant.

\section{Results}

\section{Macroscopic and Microscopic Findings of the Coronary Section of the Femoral Head}

The resected femoral heads were fixed with $10 \%$ formalin and then decalcified in 10\% EDTA-2Na solution. Several months later, the presence or absence of necrosis in the coronary section of the femoral head was determined macroscopically. Overt necrosis was observed in the coronary section of the femoral heads derived from idiopathic ONFH patients (Figure 1A). Necrosis was not apparent in the coronary section of the femoral heads derived from OA patients (Figure 1C). Afterward, these macroscopic findings were confirmed microscopically (Figure 1, B and D).

Table 2 Existence of NET-Forming Neutrophils in Small Vessels Surrounding the Femoral Head

\begin{tabular}{lccc}
\hline \multirow{2}{*}{ Patients } & \multicolumn{2}{l}{ NET-forming neutrophils } & \\
\cline { 2 - 3 } & Absent, $n$ & Present, $n$ & $P$ value \\
\hline Idiopathic ONFH & 0 & 14 & $3.4 \times 10^{-7}$ \\
OA & 12 & 0 & \\
\hline
\end{tabular}

NET, neutrophil extracellular trap; $0 \mathrm{~A}$, osteoarthritis; $0 \mathrm{NFH}$, osteonecrosis of the femoral head. 

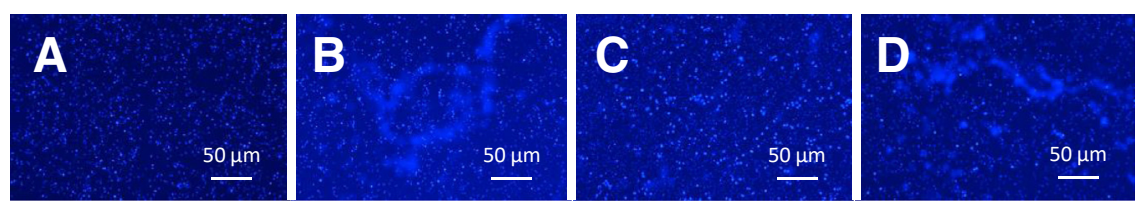

E
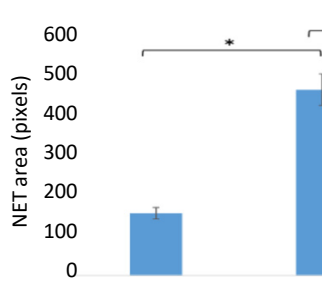

PMA $100 \mathrm{nmol} / \mathrm{L}$

$10 \%$ serum



$+$ Senior
aged
Figure 3 Serum neutrophil extracellular trap (NET) degradation assay. A-D: Representative microphotographs: $100 \mathrm{nmol} / \mathrm{L}$ phorbol 12myristate 13 -acetate $(\mathrm{PMA} ;-), 10 \%$ serum $(-)$ $($ A); $100 \mathrm{nmol} / \mathrm{L}$ PMA $(+), 10 \%$ serum $(-)(\mathbf{B})$; $100 \mathrm{nmol} / \mathrm{L}$ PMA $(+), 10 \%$ serum of young adult (4-week-old) rats (C); and $100 \mathrm{nmol} / \mathrm{L}$ PMA (+), $10 \%$ serum of senior aged (24-week-old) rats (D). E: Area of NETs determined using ImageJ software version 1.50i. Data are expressed as means \pm SD (E). ${ }^{*} P<0.05$. Scale bars $=50 \mu \mathrm{m}(\mathbf{A}-\mathbf{D})$.

\section{NET-Forming Neutrophils in Small Vessels Surrounding the Femoral Head}

The connective tissue containing the synovial membrane appended to the femoral head (Figure 2A) was sampled before decalcifying the bone and subjected to hematoxylin and eosin staining and immunofluorescence staining for NET-forming neutrophils. In all 14 cases of idiopathic ONFH, NET-forming neutrophils identified by a colocalization of citrullinated histone H3 (NET marker ${ }^{14}$ ), MPO, and DAPI (DNA marker) were found in small vessels surrounding the femoral head (Figure 2B), whereas no thrombus was found in the hematoxylin and eosin specimens. In contrast, $\mathrm{MPO}^{+}$cells but not NET-forming neutrophils were observed in small vessels surrounding the femoral head in all 12 cases of OA (Figure 2C). There was a statistically significant difference in the presence of NETforming neutrophils in small vessels surrounding the femoral head between idiopathic ONFH and OA $\left(\chi^{2}\right.$ test, $\left.P=3.4 \times 10^{-7}\right)($ Table 2$)$.

\section{Serum NET Degradation Ability Is Lower in Senior Aged Rats than in Young Adult Rats}

The serum NET degradation ability between young adult (4week-old) and senior aged (24-week-old) WKY rats were compared. In brief, NETs induced on human peripheral blood neutrophils by PMA were exposed to $10 \%$ rat serum for 30 minutes at $37^{\circ} \mathrm{C}$, and then residual NETs were quantified by image analysis. As a result, the serum NET degradation ability of senior aged rats was found to be significantly lower than that of young adult rats (Figure 3).


Figure 4 Circulation of neutrophil extracellular trap (NET)-forming neutrophils into the tissue around the femoral head of Wistar Kyoto (WKY) rats given i.v. injection of NET-forming neutrophils. A: Representative findings of NET-forming neutrophils in the tissue around the femoral head of WKY rats given i.v. injection of NET-forming neutrophils (group 1). A cluster of NET-forming neutrophils is enclosed by the dotted line. B: Representative findings of WKY rats given i.v. injection of neutrophils without NET induction (group 2). Myeloperoxidase-positive (MPO ${ }^{+}$) cells (arrowheads) but not NET-forming neutrophils are observed. Scale bars $=50 \mu \mathrm{m}(\mathbf{A}$ and $\mathbf{B})$. Cit H3, citrullinated histone $\mathrm{H} 3$. 
Table 3 Existence of NET-Forming Neutrophils in Small Vessels Surrounding the Femoral Head

\begin{tabular}{llll}
\hline & \multicolumn{2}{l}{$\begin{array}{l}\text { NET-forming } \\
\text { neutrophils }\end{array}$} & \\
\cline { 2 - 3 } Rat group & Absent & Present & $P$ value \\
\hline Legs of group 1 & 4 & 8 & 0.0029 \\
Legs of group 2 & 8 & 0 & \\
\hline
\end{tabular}

group 1, Wistar Kyoto (WKY) rats given i.v. injection of neutrophil extracellular trap (NET)-forming neutrophils; group 2, WKY rats given i.v. injection of neutrophils without NET induction.

\section{Circulation of NET-Forming Neutrophils into the Tissue Around the Femoral Head of Rats Intravenously Injected with NET-Forming Neutrophils}

According to the results of the serum NET degradation assay, NET-forming neutrophils were injected into senior aged (24-week-old) WKY rats (group $1 ; n=6$ ) via the tail vein (substance derived from $2 \times 10^{7}$ cells/kg recipient weight) twice with 1-week interval. For this purpose, cells collected from the abdominal cavity of WKY rats with peritonitis were stimulated by PMA to induce NETs in culture dishes. After removal of PMA by washing with PBS, the residual substance that contained abundant NET-forming neutrophils was injected into rats. As controls,

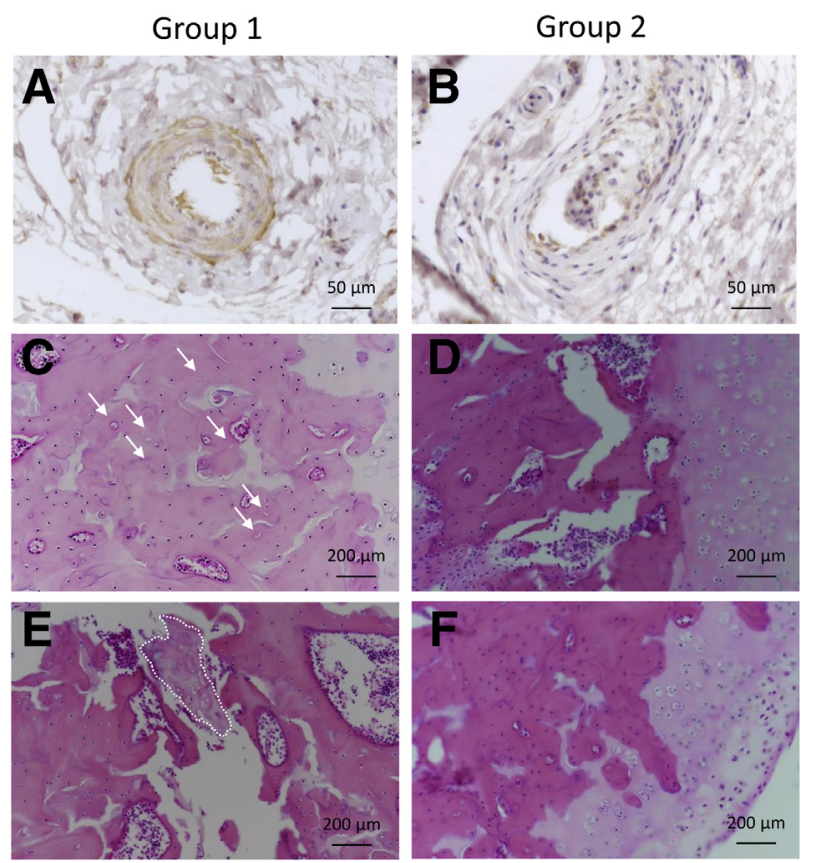

Figure 5 Ischemic state of the femoral head. The femoral heads and surrounding tissue of Wistar Kyoto (WKY) rats given i.v. injection of neutrophil extracellular trap (NET)-forming neutrophils (group 1; A, C, and E) and WKY rats given i.v. injection of neutrophils without NET induction (group 2; B, D, and F) were subjected to immunohistochemistry for hypoxia-inducible factor- $1 \alpha(\mathbf{A}$ and $\mathbf{B})$ and hematoxylin and eosin staining $(\mathbf{C}-\mathbf{F})$. Representative findings are shown. White arrows indicate osteocytes with disappeared nuclei. The area enclosed by the dotted line exhibits a necrotic lesion. Scale bars: $50 \mu \mathrm{m}$ (A and B); $200 \mu \mathrm{m}(\mathbf{C}-\mathbf{F})$.
Table 4 Ischemic Change of Osteocytes in the Femoral Head

\begin{tabular}{llll}
\hline & \multicolumn{2}{l}{$\begin{array}{l}\text { Ischemic change of } \\
\text { osteocytes }\end{array}$} & \\
\cline { 2 - 3 } Rat group & Absent, $n$ & Present, $n$ & $P$ value \\
\hline Legs of group 1 & 10 & 2 & 0.22 \\
Legs of group 2 & 8 & 0 & \\
\hline
\end{tabular}

group 1, Wistar Kyoto (WKY) rats given i.v. injection of neutrophil extracellular trap (NET)-forming neutrophils; group 2, WKY rats given i.v. injection of neutrophils without NET induction.

the cells collected from the abdominal cavity of rats with peritonitis were similarly injected into senior aged (24-week-old) WKY rats (group 2; $n=4 ; 2 \times 10^{7}$ cells $/ \mathrm{kg}$ recipient weight) without NET induction. In group 1 , NET-forming neutrophils were found in small vessels at either the left or the right connective tissue around the femoral head in all 6 rats (8/12 legs) (Figure 4A). In group $2, \mathrm{MPO}^{+}$cells but not NET-forming neutrophils were observed in small vessels surrounding the femoral head $(0 / 8$ legs) (Figure 4B). There was a statistically significant difference in the presence of NET-forming neutrophils in small vessels surrounding the femoral head between groups 1 and $2\left(\chi^{2}\right.$ test, $\left.P=0.0029\right)$ (Table 3$)$.

\section{Ischemic Change of the Femoral Head of Rats Intravenously Injected with NET-Forming Neutrophils}

To determine the ischemic state of the femoral head of WKY rats intravenously injected with NET-forming neutrophils, the expression of HIF- $1 \alpha$ in the connective tissue appended to the femoral head was examined. Results demonstrated that HIF-1 $\alpha$ expression in vascular smooth muscle cells in the connective tissue around the femoral head of group 1 was higher compared with that of group 2 (Figure 5, A and B). Correspondingly, ischemic change of osteocytes in the femoral head represented by a decrease in nuclear staining for hematoxylin was observed in two legs of two rats in group 1 (2/12 legs) (Figure 5, C and E), although no thrombus was found in the connective tissue around the femoral head. Ischemic change of osteocytes in the femoral head was not apparent in group 2 (0/8 legs) (Figure 5, D and F). Although there was no statistically significant difference between groups 1 and 2 ( $\chi^{2}$ test, $P=0.22$ ) (Table 4 ), the collective findings suggested the association of NET-forming neutrophils in small vessels surrounding the femoral head with ischemia of the femoral head.

\section{Discussion}

In this study, we first demonstrated the presence of NETforming neutrophils in small vessels surrounding the femoral head of patients with idiopathic ONFH but not OA. It has been considered that ONFH is developed consequently to a compromised subchondral microcirculation. ${ }^{16}$ 
Because of the limitation of method-immunofluorescence staining for NETs prefers tissue sections without decalcification, small vessels were assessed in the connective tissue surrounding the femoral head instead of the subchondral small vessels in the bone. Results indicate the association of idiopathic ONFH and the presence of NET-forming neutrophils in small vessels near to the lesion. However, it is elusive whether NET-forming neutrophils are involved in the development of idiopathic ONFH or are present there as a result of the disease. Therefore, animal experiments were then planned.

Diverse animal models of idiopathic ONFH, including glucocorticoid-induced ONFH in rats, mice, rabbits, chickens, pigs, and emus (reviewed by $\mathrm{Xu}$ et $\mathrm{al}^{17}$ ) and alcohol-induced ONFH in rats, ${ }^{18}$ goats,,${ }^{19}$ and canine, ${ }^{20}$ have been established. Although these models mimic the clinical characteristics of idiopathic ONFH, we contrived a simple induction model to determine the direct contribution of NET-forming neutrophils to the development of ONFH. For this purpose, inbred wild-type WKY rats that are often used for vascular research focusing on vasodynamics ${ }^{21}$ and vasculitides were employed. ${ }^{22}$

NET-forming neutrophils intravenously injected into WKY rats circulated into the tissue around the femoral head, and the expression of HIF- $1 \alpha$ in the tissue was higher compared with the control tissue. Correspondingly, ischemic change of osteocytes in the femoral head, represented by a decrease in nuclear staining for hematoxylin, was observed in some rats given an i.v. injection of NET-forming neutrophils. It has been demonstrated that the expression of HIF- $1 \alpha$ is induced by tissue hypoxia. ${ }^{23}$ On the basis of these findings, we consider that NETs are possibly involved in the local blood flow disturbance and ischemia of the femoral head. However, factors other than NETs could be also involved in the pathogenesis of ONFH because an apparent necrosis of osteocytes that resulted in the destruction of the femoral head was not observed in the rats.

Overt thrombi have not been found in the connective tissue around the femoral head of either patients with ONFH or the rats given an i.v. injection of NET-forming neutrophils. Although NETs link thrombosis, ${ }^{24}$ mechanisms other than thrombogenesis (eg, spasm of small vessels with NET-mediated injury of endothelial cells) may be involved in the tissue ischemia. Further studies are needed to determine how NET-forming neutrophils in small vessels contribute to tissue ischemia.

NET-forming neutrophils do not seem to be recruited into the tissue around the femoral head site specifically, because the deposition of NETs in the small vessel wall has been also observed in other sites of connective tissues in the rats (Supplemental Figure S1). These findings suggest that the intravenously injected NET-forming neutrophils are circulating in the body of rats. However, the possibility that a certain anatomic factor is associated with the local blood flow disturbance of the femoral head cannot be denied. ${ }^{25}$
In this study, cells exhibiting a colocalization of citrullinated histone H3, MPO, and DAPI were regarded as NETforming neutrophils. However, these cells may include extracellular trap-forming monocytes, as monocytes-which also contain MPO-have been shown to form extracellular traps similarly to neutrophils. ${ }^{26}$ Additional studies are needed to identify citrullinated histone $\mathrm{H} 3-$ positive, $\mathrm{MPO}^{+}$, and DAPI $^{+}$cells as NET-forming neutrophils.

The amount of NETs in vivo is estimated as a balance of generation and degradation. The generation of NETs is physiologically induced by infectious stimuli, such as lipopolysaccharides. ${ }^{8}$ Under pathologic conditions, diverse factors that activate neutrophils, including inflammatory cytokines and autoantibodies, can induce NETs. ${ }^{27}$ These NET-inducible factors are generally associated with autoimmune diseases, which are the targets of glucocorticoid treatment. In addition, it has been shown that the number of lowdensity granulocytes, which are apt to generate NETs, is increased in patients with autoimmune diseases, including systemic lupus erythematosus. ${ }^{28}$ Therefore, it is considered that patients who developed glucocorticoid-associated ONFH have been liable to generate NETs because of underlying diseases. Further studies are needed to clarify how glucocorticoid enhances NET formation on neutrophils of patients with diverse autoimmune diseases. Bukong et $\mathrm{al}^{11}$ have recently demonstrated that alcohol can induce NETs in vitro. Thus, it is also considered that patients who developed alcohol-associated ONFH have been liable to generate NETs.

The two major mechanisms of NET degradation are digestion by serum DNase $\mathrm{I}^{12}$ and efferocytosis by macrophages. ${ }^{29}$ The low ability of NET degradation in the sera of patients with autoimmune diseases, including systemic lupus erythematosus ${ }^{12}$ and microscopic polyangiitis, ${ }^{30}$ has been demonstrated. Moreover, the impairment of efferocytosis in systemic lupus erythematosus has been shown. ${ }^{31}$ Interestingly, alcohol binges induced a reduction of efferocytosis by macrophages in mice. ${ }^{11}$ The determination of NET degradation disorder in patients with idiopathic ONFH is a critical subject in future studies.

\section{Conclusions}

Idiopathic ONFH develops in patients with a predisposing genetic background who have been exposed to causative environmental factors. This study suggests the association of NETs with the development of idiopathic ONFH and promotes the understanding of the pathophysiology.

\section{Author Contributions}

T.S., S.M., D.N., U.T., and A.I. conceived the study, designed the experiments, and analyzed data; M.N., T.S., M.Y., Y.H., Y.N., D.T., T.A., Y.N., S.M., and A.I. performed the experiments; M.N., T.S., S.M., D.N., S.T., U.T., 
N.I., and A.I. discussed results; all authors read and approved the final version of the manuscript.

\section{Supplemental Data}

Supplemental material for this article can be found at http://doi.org/10.1016/j.ajpath.2020.07.008.

\section{References}

1. Mankin HJ: Nontraumatic necrosis of bone (osteonecrosis). N Engl J Med 1992, 326:1473-1479

2. Petek D, Hannouche D, Suva D: Osteonecrosis of the femoral head: pathophysiology and current concepts of treatment. EFORT Open Rev 2019, 4:85-97

3. Kubo T, Ueshima K, Saito M, Ishida M, Arai Y, Fujiwara H: Clinical and basic research on steroid-induced osteonecrosis of the femoral head in Japan. J Orthop Sci 2016, 21:407-413

4. Wang A, Ren M, Wang J: The pathogenesis of steroid-induced osteonecrosis of the femoral head: a systematic review of the literature. Gene 2018, 671:103-109

5. Zhang Q, Jin LV, Jin L: Role of coagulopathy in glucocorticoid-induced osteonecrosis of the femoral head. J Int Med Res 2018, 46:2141-2148

6. Hirota Y, Hirohata T, Fukuda K, Mori M, Yanagawa H, Ohno Y, Sugioka Y: Association of alcohol intake, cigarette smoking, and occupational status with the risk of idiopathic osteonecrosis of the femoral head. Am J Epidemiol 1993, 137:530-538

7. Liu YF, Chen WM, Lin YF, Yang RC, Lin MW, Li LH, Chang YH, Jou YS, Lin PY, Su JS, Huang SF, Hsiao KJ, Fann CS, Hwang HW, Chen YT, Tsai SF: Type II collagen gene variants and inherited osteonecrosis of the femoral head. N Engl J Med 2005, 352: 2294-2301

8. Brinkmann V, Reichard U, Goosmann C, Fauler B, Uhlemann Y, Weiss DS, Weinrauch Y, Zychlinsky A: Neutrophil extracellular traps kill bacteria. Science 2004, 303:1532-1535

9. Doring Y, Weber C, Soehnlein O: Footprints of neutrophil extracellular traps as predictors of cardiovascular risk. Arterioscler Thromb Vasc Biol 2013, 33:1735-1736

10. Zucoloto AZ, Jenne CN: Platelet-neutrophil interplay: insights into neutrophil extracellular trap (NET)-driven coagulation in infection. Front Cardiovasc Med 2019, 6:85

11. Bukong TN, Cho Y, Iracheta-Vellve A, Saha B, Lowe P, Adejumo A, Furi I, Ambade A, Gyongyosi B, Catalano D, Kodys K, Szabo G: Abnormal neutrophil traps and impaired efferocytosis contribute to liver injury and sepsis severity after binge alcohol use. J Hepatol 2018, 69:1145-1154

12. Hakkim A, Furnrohr BG, Amann K, Laube B, Abed UA, Brinkmann V, Herrmann M, Voll RE, Zychlinsky A: Impairment of neutrophil extracellular trap degradation is associated with lupus nephritis. Proc Natl Acad Sci U S A 2010, 107:9813-9818

13. Koizumi T: Tissue distribution of deoxyribonuclease I (DNase I) activity level in mice and its sexual dimorphism. Exp Anim 1995, 44: $181-185$

14. Masuda S, Shimizu S, Matsuo J, Nishibata Y, Kusunoki Y, Hattanda F, Hatanaka Y, Nakazawa D, Tanaka S, Tomaru U, Kawakami T, Atsumi T, Ishizu A: Measurement of NET formation in vitro and in vivo by flow cytometry. Cytometry A 2017, 91:822-829

15. Hattanda F, Nakazawa D, Watanabe-Kusunoki K, Kusunoki Y, Shida H, Masuda S, Nishio S, Tomaru U, Atsumi T, Ishizu A: The presence of anti-neutrophil extracellular trap antibody in patients with microscopic polyangiitis. Rheumatology (Oxford) 2019, 58:1293-1298

16. Shah KN, Racine J, Jones LC, Aaron RK: Pathophysiology and risk factors for osteonecrosis. Curr Rev Musculoskelet Med 2015, 8: 201-209

17. Xu J, Gong H, Lu S, Deasey MJ, Cui Q: Animal models of steroidinduced osteonecrosis of the femoral head-a comprehensive research review up to 2018. Int Orthop 2018, 42:1729-1737

18. Okazaki S, Nagoya S, Tateda K, Katada R, Mizuo K, Watanabe S, Yamashita T, Matsumoto H: Experimental rat model for alcoholinduced osteonecrosis of the femoral head. Int J Exp Pathol 2013, 94:312-319

19. Zhu ZH, Gao YS, Luo SH, Zeng BF, Zhang CQ: An animal model of femoral head osteonecrosis induced by a single injection of absolute alcohol: an experimental study. Med Sci Monit 2011, 17: BR97-BR102

20. Wang C, Wang J, Zhang Y, Yuan C, Liu D, Pei Y, Li X, Wu Z, Li Y, Guo Z: A canine model of femoral head osteonecrosis induced by an ethanol injection navigated by a novel template. Int J Med Sci 2013, 10:1451-1458

21. Prieto I, Segarra AB, de Gasparo M, Martinez-Canamero M, RamirezSanchez M: Divergent profile between hypothalamic and plasmatic aminopeptidase activities in WKY and SHR: influence of $\beta$-adrenergic blockade. Life Sci 2018, 192:9-17

22. Little MA, Smyth L, Salama AD, Mukherjee S, Smith J, Haskard D, Nourshargh S, Cook HT, Pusey CD: Experimental autoimmune vasculitis: an animal model of anti-neutrophil cytoplasmic autoantibody-associated systemic vasculitis. Am J Pathol 2009, 174: $1212-1220$

23. Fernandez-Torres J, Zamudio-Cuevas Y, Martinez-Nava GA, LopezReyes AG: Hypoxia-inducible factors (HIFs) in the articular cartilage: a systematic review. Eur Rev Med Pharmacol Sci 2017, 21: 2800-2810

24. Brill A, Fuchs TA, Savchenko AS, Thomas GM, Martinod K, De Meyer SF, Bhandari AA, Wagner DD: Neutrophil extracellular traps promote deep vein thrombosis in mice. J Thromb Haemost 2012, 10: $136-144$

25. Ollivier M, Lunebourg A, Abdel MP, Parratte S, Argenson JN: Anatomical findings in patients undergoing total hip arthroplasty for idiopathic femoral head osteonecrosis. J Bone Joint Surg Am 2016, 98: $672-676$

26. Granger V, Faille D, Marani V, Noel B, Gallais Y, Szely N, Flament H, Pallardy M, Chollet-Martin S, de Chaisemartin L: Human blood monocytes are able to form extracellular traps. J Leukoc Biol 2017, 102:775-781

27. Goel RR, Kaplan MJ: Deadliest catch: neutrophil extracellular traps in autoimmunity. Curr Opin Rheumatol 2020, 32:64-70

28. Carmona-Rivera C, Kaplan MJ: Low-density granulocytes: a distinct class of neutrophils in systemic autoimmunity. Semin Immunopathol 2013, 35:455-463

29. Gregoire M, Uhel F, Lesouhaitier M, Gacouin A, Guirriec M, Mourcin F, Dumontet E, Chalin A, Samson M, Berthelot LL, Tissot A, Kerjouan M, Jouneau S, Le Tulzo Y, Tarte K, Zmijewski JW, Tadie JM: Impaired efferocytosis and neutrophil extracellular trap clearance by macrophages in ARDS. Eur Respir J 2018, 52:1702590

30. Nakazawa D, Shida H, Tomaru U, Yoshida M, Nishio S, Atsumi T, Ishizu A: Enhanced formation and disordered regulation of NETs in myeloperoxidase-ANCA-associated microscopic polyangiitis. J Am Soc Nephrol 2014, 25:990-997

31. Kawano M, Nagata S: Efferocytosis and autoimmune disease. Int Immunol 2018, 30:551-558 